Acta Crystallographica Section D

\section{Biological \\ Crystallography}

ISSN 0907-4449

Erika V. Soriano, ${ }^{\text {a Diane E. }}$ McCloskey, ${ }^{\mathrm{b}}$ Cynthia Kinsland, ${ }^{\mathrm{a}}$ Anthony E. Pegg ${ }^{b}$ and Steven E. Ealick ${ }^{\mathrm{a} *}$

a Department of Chemistry and Chemical Biology, Cornell University, Ithaca, NY 14850-1301, USA, and ${ }^{\mathbf{b}}$ Department of Cellular and Molecular Physiology, Pennsylvania State University, College of Medicine, Hershey, PA 17033, USA

Correspondence e-mail: see3@cornell.edu

\title{
Structures of the N47A and E109Q mutant proteins of pyruvoyl-dependent arginine decarboxylase from Methanococcus jannaschii
}

Pyruvoyl-dependent arginine decarboxylase (PvlArgDC) catalyzes the first step of the polyamine-biosynthetic pathway in plants and some archaebacteria. The pyruvoyl group of PvlArgDC is generated by an internal autoserinolysis reaction at an absolutely conserved serine residue in the proenzyme, resulting in two polypeptide chains. Based on the native structure of PvlArgDC from Methanococcus jannaschii, the conserved residues Asn47 and Glu109 were proposed to be involved in the decarboxylation and autoprocessing reactions. N47A and E109Q mutant proteins were prepared and the three-dimensional structure of each protein was determined at $2.0 \AA$ resolution. The N47A and $\mathrm{E} 109 \mathrm{Q}$ mutant proteins showed reduced decarboxylation activity compared with the wild-type PvlArgDC. These residues may also be important for the autoprocessing reaction, which utilizes a mechanism similar to that of the decarboxylation reaction.

\section{Introduction}

Polyamines are essential for cell growth and proliferation in all forms of life. The polyamines spermidine and spermine have been linked to chromatin stabilization, ion-channel modulation and increased RNA translational efficiency. The relationship of polyamines to normal cell growth and proliferation suggests that their biosynthetic enzymes are potential drug targets for proliferative diseases (Tabor \& Tabor, 1984; Williams, 1997). The polyamine-biosynthetic pathway is also a target for antiparasitic agents because of the unique metabolic requirements of parasitic organisms.

In most organisms, polyamine biosynthesis requires the conversion of ornithine to putrescine by ornithine decarboxylase; however, in some bacteria and plants putrescine is generated by the decarboxylation of arginine to form agmatine by arginine decarboxylase, followed by the conversion of agmatine to putrescine by agmatine ureohydrolase (Tabor \& Tabor, 1984). In all organisms decarboxylated $S$-adenosylmethionine, which is generated by $S$-adenosylmethionine decarboxylase, serves as an aminopropyl-group donor to synthesize spermidine from putrescine and spermine from spermidine (Fig. 1).

Three classes of arginine decarboxylase exist. Two classes utilize the cofactor pyridoxal $5^{\prime}$-phosphate, while the third class uses a pyruvoyl group (Grishin \& Phillips, 1994; Sandmeier et al., 1994; Graham et al., 2002). Pyruvoyl-dependent arginine decarboxylase (PvlArgDC) is translated as a proenzyme ( $\pi$ chain). The proenzyme then undergoes autocatalytic serinolysis, resulting in the formation of two chains and the creation of a pyruvoyl group, which is the cofactor for the decarboxylation reaction. By convention, the $\beta$ subunit
Received 18 July 2007

Accepted 7 January 2008

PDB References: pyruvoyldependent arginine decarboxylase, E109Q mutant, 2qqc, r2qqcsf; N47A mutant, 2qqd, r2qqdsf. 
consists of the N-terminal fragment, while the $\alpha$ subunit contains the C-terminal fragment with the pyruvoyl group at its N-terminus (Recsei et al., 1983; van Poelje \& Snell, 1990). In Methanococcus jannaschii PvlArgDC serinolysis occurs between Ser52 and Ser53, resulting in a $\beta$ subunit consisting of 52 amino acids and an $\alpha$ subunit of 113 amino acids (Graham et al., 2002).

To understand the mechanism of the autocatalytic serinolysis, two mutant proteins, E109Q and N47A, were prepared based on analysis of the wild-type PvlArgDC structure (Tolbert et al., 2003). Glu109 was proposed to act as an acid in the formation of the ester intermediate and a base during the $\beta$-elimination step to form the pyruvoyl group. Asn47 is believed to play a structural role and is widely conserved not only in PvlArgDC but also in the structurally related pyruvoyl-dependent histidine decarboxylase (Tolbert et al., 2003). We determined the crystal structures of PvlArgDC N47A and E109Q mutant proteins to $2.0 \AA$ resolution and compared the structures with that of wild-type PvlArgDC.

\section{Experimental procedures}

\subsection{Mutagenesis of PRDC: molecular cloning}

Standard methods were used for DNA manipulations (Ausubel et al., 1987; Sambrook et al., 1989). Plasmid DNA was purified with a Qiagen Miniprep kit. Escherichia coli strain MachI (Invitrogen) was used as a recipient for transformations during plasmid construction and for plasmid propagation and storage. Site-directed mutagenesis was performed on pPRDC.19 (as described previously; Tolbert et al., 2003) by a PCR protocol using $P f u$ Turbo DNA polymerase as per the manufacturer's instructions (Invitrogen) and DpnI (New England Biolabs) to digest the methylated parental DNA prior to transformation.

For the E109Q mutant protein, the following complementary primer pair was used: PRDCE109QF, 5'-GAG TTT ATG TGG TTT AAT AAT GCA GTA CGA AGG AAA ATG CTC-3', and PRDCE109QR，5'-GAG CAT TTT CCT TCG TAC TGC ATT ATT AAA CCA CAT AAA CTC-3'. Clones were screened by restriction digest for the introduction of an RsaI site. A representative clone with the correct restriction pattern was sequenced and named pPRDC.19.E109Q.
For the N47A mutant protein, the following complementary primer pair was used: PRDCN47AF, 5'-GCA GGC ATA GGG AAT GTC GCA TTA ATT AGA ATC AGC AGT ATA ATG CC-3', and PRDCN47AR, 5'-GGC ATT ATA CTG CTG ATT CTA ATT AAT GCG ACA TTC CCT ATG CCT GC-3'. Clones were screened by restriction digest for the introduction of an AseI site. A representative clone with the correct restriction pattern was sequenced and named pPRDC.19.N47A.

\subsection{Protein expression and purification}

The pPRDC.19.E109Q and pPRDC.19.N47A plasmids were each transformed into E. coli B834 (DE3) competent cells. Expression, purification and cleavage of the N-terminal His tag were performed as described previously (Tolbert et al.,<smiles>C[S+](CCC([NH3+])C(=O)[O-])CC(O)OC(O)n1cnc2c(N)ncnc21</smiles>

$S$-Adenosylmethionine
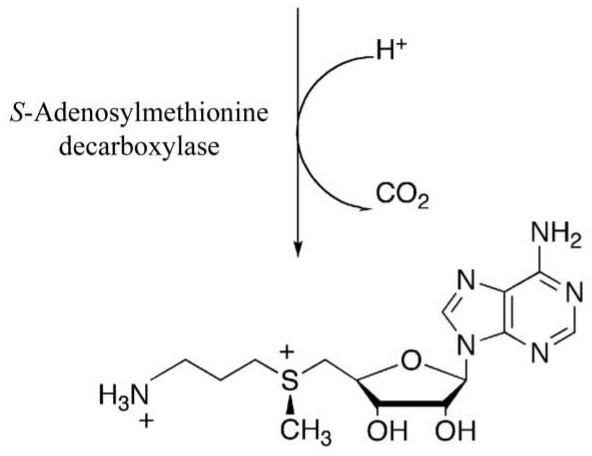

Decarboxylated $S$-adenosylmethionine

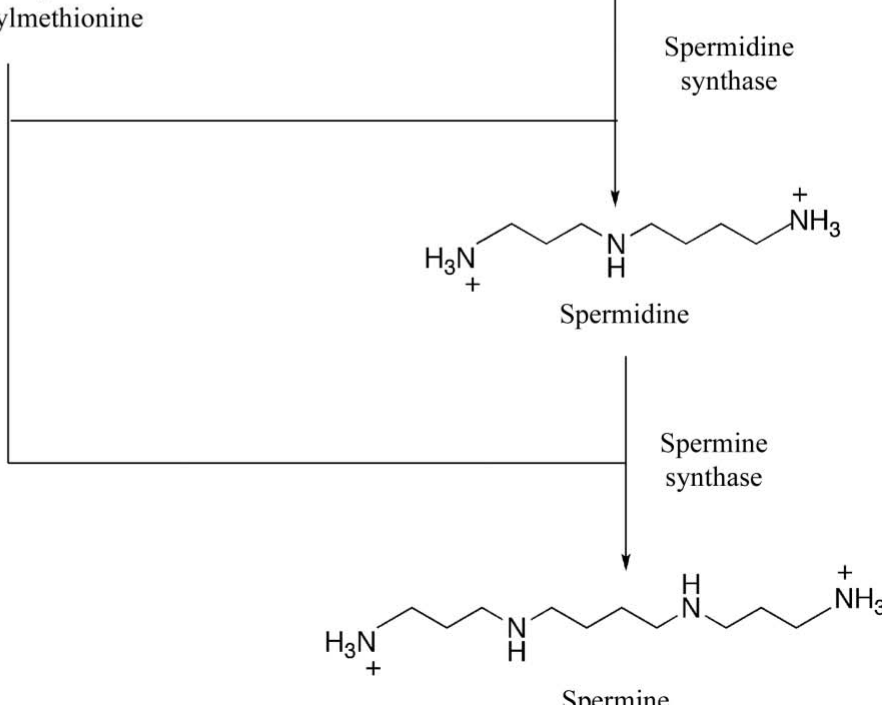

Figure 1

The PvlArgDC-based polyamine-biosynthetic pathway. In most organisms putrescine is produced by the decarboxylation of ornithine by ornithine decarboxylase. 
Table 1

Data-collection and refinement statistics for PvlArgDC mutant proteins.

Values in parentheses are for the highest resolution bin.

\section{PvlArgDC E109Q PvlArgDC N47A}

\begin{tabular}{|c|c|c|}
\hline \multicolumn{3}{|l|}{ Data-collection statistics } \\
\hline Resolution $(\AA)$ & 2.0 & 2.0 \\
\hline No. of reflections & 247483 & 222205 \\
\hline No. of unique reflections & 60058 & 58029 \\
\hline Redundancy & $4.1(3.4)$ & $3.8(2.9)$ \\
\hline Completeness (\%) & $98.3(97.8)$ & $95.5(83.3)$ \\
\hline$R_{\text {merge }} \dagger(\%)$ & $5.0(9.5)$ & $9.2(31.3)$ \\
\hline$I / \sigma(I)$ & $20.9(13.5)$ & $16.8(4.0)$ \\
\hline \multicolumn{3}{|l|}{ Refinement statistics } \\
\hline Resolution $(\AA)$ & $50-2.0$ & $50-2.0$ \\
\hline$R$ factor $(\%)$ & 19.4 & 21.3 \\
\hline$R_{\text {free }} \S(\%)$ & 23.5 & 25.9 \\
\hline \multicolumn{3}{|l|}{ Total no. of non-H atoms } \\
\hline Protein atoms & 7144 & 7135 \\
\hline Water atoms & 524 & 364 \\
\hline Ligand/MPD atoms & 94 & 51 \\
\hline \multicolumn{3}{|l|}{$B$ factors $\left(\AA^{2}\right)$} \\
\hline Protein atoms & 22.8 & 31.2 \\
\hline Water atoms & 33.5 & 30.8 \\
\hline Ligand/MPD atoms & 39.4 & 59.0 \\
\hline \multicolumn{3}{|l|}{ R.m.s. deviations } \\
\hline Bonds $(\AA)$ & 0.005 & 0.006 \\
\hline Angles $\left({ }^{\circ}\right)$ & 1.29 & 1.38 \\
\hline \multicolumn{3}{|l|}{ Ramachandran plot } \\
\hline Most favored regions (\%) & 90.4 & 88.6 \\
\hline Additional allowed regions (\%) & 9.6 & 11.4 \\
\hline Disallowed regions (\%) & 0.0 & 0.0 \\
\hline
\end{tabular}

$\dagger R_{\text {merge }}=\sum_{h k l} \sum_{i}\left|I_{i}(h k l)-\langle I(h k l)\rangle\right| / \sum_{h k l} \sum_{i} I_{i}(h k l)$, where $\langle I(h k l)\rangle$ is the mean intensity of the $i$ reflections with intensity $I(h k l)$ and common indices $h k l$. $\$ R$ factor $=$ $\sum_{h k l}|| F_{\text {obs }}|-| F_{\text {calc }}|| / \sum_{h k l}\left|F_{\text {obs }}\right|$, where $F_{\text {obs }}$ and $F_{\text {calc }}$ are observed and calculated structure factors, respectively. $\S$ For $R_{\text {free }}$, the sum is extended over a subset of reflections $(10 \%)$ excluded from all stages of refinement.

2003). The protein was buffer-exchanged to $50 \mathrm{~m} M$ Tris- $\mathrm{HCl}$ $\mathrm{pH} 8.5,250 \mathrm{~m} M \mathrm{NaCl}$ and $1 \mathrm{~m} M$ dithiothreitol (DTT) and concentrated to $10 \mathrm{mg} \mathrm{ml}^{-1}$. The cleaved samples were stored at $277 \mathrm{~K}$ until use in crystallization trials.

\subsection{Crystallization}

Both the N47A and E109Q mutant proteins were cocrystallized at $295 \mathrm{~K}$ with $1-2 \mathrm{~m} M$ arginine. The hanging-drop vapor-diffusion method was used. The drop contained $1 \mu \mathrm{l}$ well solution and $1 \mu \mathrm{l}$ protein solution. The crystallization conditions for both mutant proteins are similar to those used for the wild-type protein (Tolbert et al., 2003). The crystals were grown in 17-20\%(w/w) PEG 2000, 10\% 2-methyl2,4-pentanediol (MPD), 2.5\% glycerol, $100 \mathrm{~m} M$ HEPES $\mathrm{pH}$ 6.7-7.3, $0.5 \mathrm{~m} M \quad \beta$-octylglucoside, $0.5 \mathrm{~m} M$ ethylenediaminetetraacetic acid and $10 \mathrm{~m} M$ DTT. The crystals were frozen directly in the cryostream prior to data collection without additional cryoprotectant. Both mutant proteins produced crystals belonging to space group $P 2_{1}$. The unit-cell parameters were $a=57.7, b=92.2, c=86.1 \AA, \beta=94.5^{\circ}$ for the N47A mutant and $a=58.1, b=92.8, c=85.7 \AA, \beta=95.1^{\circ}$ for the E109Q mutant.

\subsection{Data collection and structure determination}

X-ray diffraction data were collected on the A1 beamline at the Cornell High Energy Synchrotron Source (CHESS) using a Q210 detector (Area Detector Systems Corporation) in binned mode. The data were collected with an oscillation of $0.5^{\circ}$ at a wavelength of $0.9760 \AA$. The data were indexed, integrated and scaled with HKL-2000 (Otwinowski \& Minor, 1997). Data-collection statistics are given in Table 1.

The structures of the N47A and E109Q mutant proteins were determined by molecular replacement using $C N S$ (Brünger et al., 1998) with the wild-type PvlArgDC as the starting model (PDB code 1n13). Model building was performed using the programs $O$ (Jones et al., 1991) and Coot (Emsley \& Cowtan, 2004), with the program CNS used for refinement. PROCHECK (Laskowski et al., 1993) was used to determine the quality of the final model. Final data-refinement statistics are shown in Table 1.

\subsection{Activity assays}

The E109Q and N47A mutant proteins were assayed for decarboxylation activity based on a procedure described by Graham et al. (2002). The PvlArgDC activity of each mutant was determined by measuring the rate of ${ }^{14} \mathrm{CO}_{2}$ production using ${ }^{14} \mathrm{C}$-arginine as the substrate. The assays were carried out at $343 \mathrm{~K}$ for $30 \mathrm{~min}$ in $50 \mathrm{~m} M 2-(N$-morpholino $)$ ethanesulfonic acid/NaOH buffer $\mathrm{pH}$ 6.0, $50 \mathrm{~m} M \mathrm{KCl}, 1 \mathrm{~m} M$ DTT with $4 \mathrm{~m} M_{\mathrm{L}}$-arginine and $2 \mu \mathrm{l}^{14} \mathrm{C}_{\mathrm{L}}$-arginine. The reaction was terminated by the addition of $\mathrm{HCl}$. The level of ${ }^{14} \mathrm{CO}_{2}$ was measured using a scintillation counter. The specific activity of the enzyme was then calculated based on the amount of ${ }^{14} \mathrm{CO}_{2}$ released.

Efforts to measure the rate of autoprocessing were inconclusive (unpublished results). The mutant proteins showed little processing after $3 \mathrm{~h}$ at $338 \mathrm{~K}$; however, the wild-type protein failed to dissociate to the monomeric form and therefore the relative rates of autoprocessing could not be determined.

\subsection{Figure preparation}

All figures were generated using MolScript (Esnouf, 1997, 1999), Raster3D (Merritt \& Bacon, 1997) and PyMOL (DeLano, 2002).

\section{Results and discussion}

\subsection{Structures of the PvlArgDC mutant proteins}

The structures of both PvlArgDC mutant proteins contain two complete trimers in the asymmetric unit. The active sites of each trimer are located between adjacent protomers. The protomer (Fig. 2) is a four-layer $\alpha \beta \beta \alpha$ sandwich as described previously (Tolbert et al., 2003). For the E109Q mutant protein, all six protomers were fully processed and contained the product agmatine at the active site. The presence of the product agmatine confirms that the E109Q mutant is active 

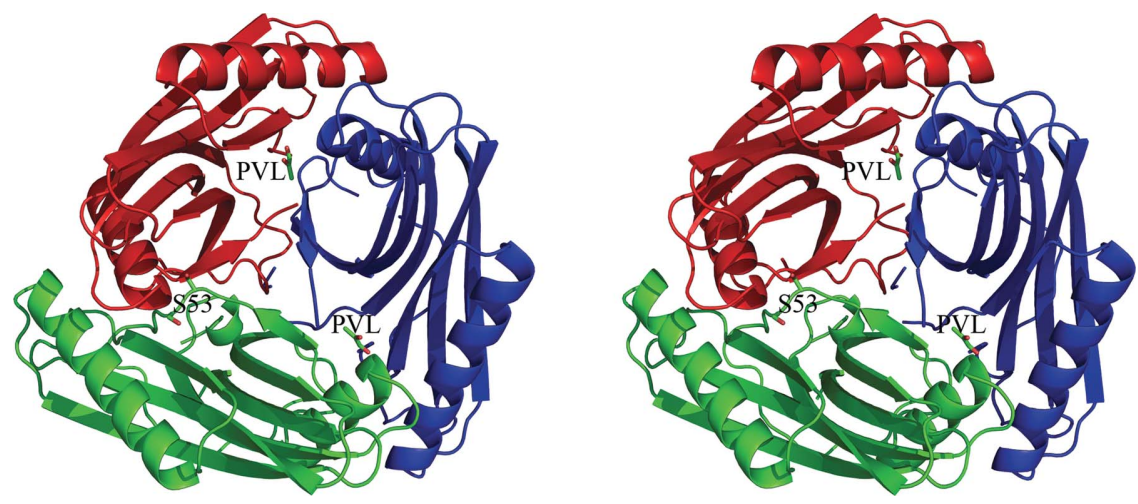

Figure 2

Ribbon diagram of the N47A PvlArgDC trimer colored by protomer. The pyruvoyl cofactor is shown in ball-and-stick representation and is found in the red and blue protomers. The third protomer is unprocessed, with Ser53 shown in ball-and-stick representation. The atoms are colored by element: green for carbon, red for oxygen and blue for nitrogen.
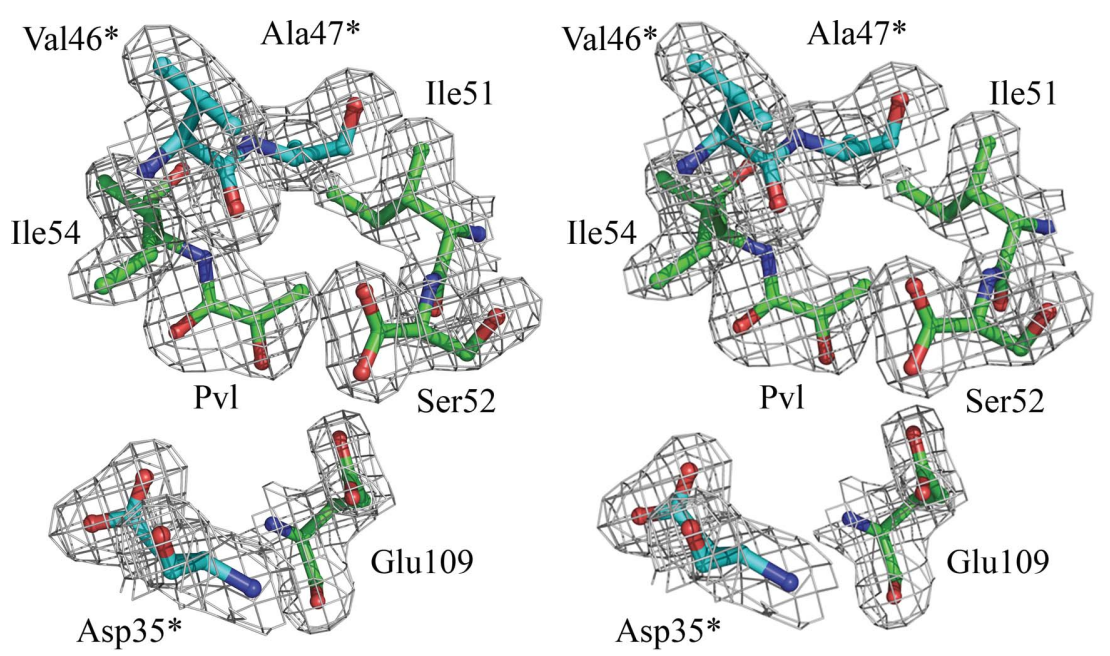

(a)
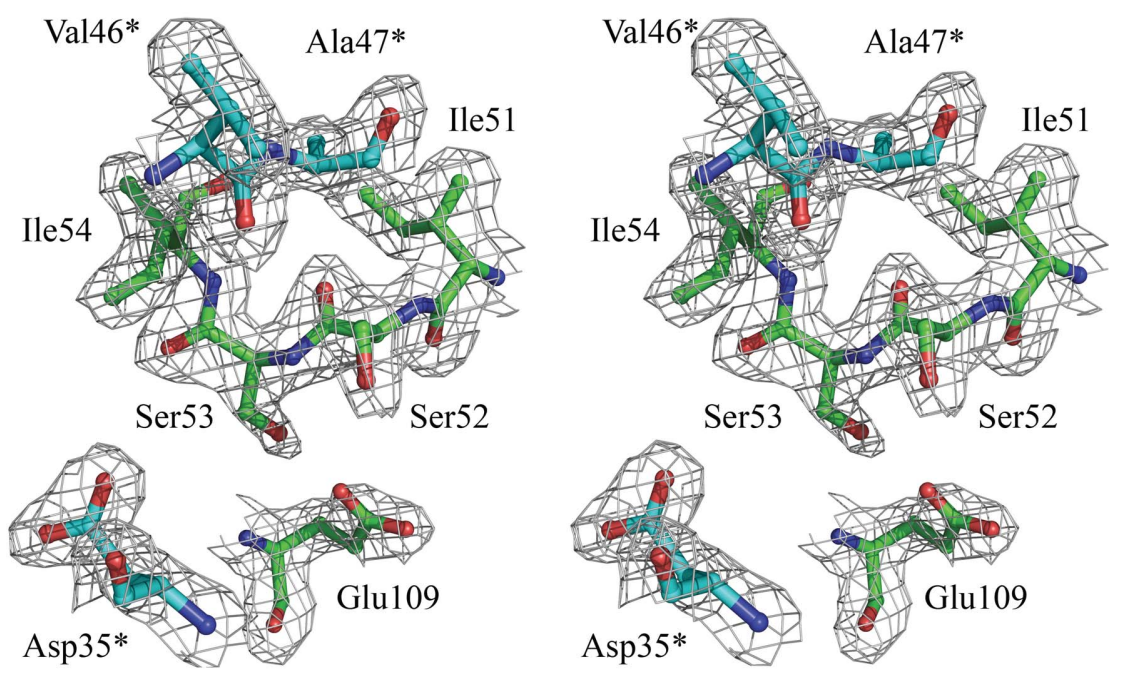

(b)

Figure 3

Stereodiagram of the N47A active sites. (a) The active sites of chains $A$ and $B$ are shown. The electron density shows that the chain is processed. $(b)$ The active site of the unprocessed monomer, chain $C$. The electron density between Ser52 and Ser53 is continuous and the sidechain density is also apparent for both residues. In all panels residues marked with an asterisk are from a second protomer. because the substrate arginine was added to the protein during crystallization. The final $R$ factor is $19.4 \%$ and $R_{\text {free }}$ is $23.5 \%$.

Unlike the E109Q mutant protein, the N47A mutant protein did not show complete processing in all protomers. The first protomer of N47A was processed, with the electron density showing clear cleavage of the protomer to form the $\beta$ chain (residues 1-52) and the $\alpha$ chain (residues 53165) (Fig. 3a). A second protomer was unprocessed, showing clear density connecting residues Ser52 and Ser53 (Fig. 3b). The final protomer in the first trimer could not easily be classified as either processed or unprocessed and is likely to be a mixture of the two states. The density between Ser52 and Ser53 was weak; however, density was also present corresponding to the pyruvoyl group and the product agmatine. Based on the density and the refined $B$ factors, the predominant form was assigned as processed for the third protomer in the first asymmetric unit. All three protomers of the second trimer were unprocessed. The final $R$ factor is $21.3 \%$ and $R_{\text {free }}$ is $25.9 \%$.

\subsection{The PvIArgDC active site}

The product of the reaction was located in all six active sites in the E109Q mutant protein and in two of the six active sites in the N47A mutant protein. The E109Q active site is similar to that of the wild-type enzyme as shown in Fig. 4(a). Superposition of the two structures resulted in an overall r.m.s.d. of $0.2 \AA^{2}$. Superimposing the two active sites revealed a change in the rotamer conformation of Gln109 when compared with Glu109 in the wild-type structure. This change in conformation allows the $\mathrm{N}^{\varepsilon 2}$ atom of $\mathrm{Gln} 109$ to form a hydrogen bond to the carbonyl $\mathrm{O}$ atom of Tyr77. In the wild-type structure, the Glu109 side chain is shifted closer to the $\mathrm{N}$ atom of agmatine, causing the side chain to be $3.7 \AA$ from the Tyr77 carbonyl. The other active-site residues superimpose well.

The N47A structure revealed a loss in hydrogen bonding from the Asn47 side chain to the main chain of Ile54 when compared with the S53A nonprocessing mutant protein structure (PDB code $1 \mathrm{n} 2 \mathrm{~m}$; Fig. 4b). This loss of a hydrogen bond results in a main-chain movement of $0.5 \AA$ in both the processed and unprocessed chains of the 
N47A structure. There is also increased flexibility around residues 51-54. A superposition of three unprocessed chains from the N47A structure showed a variety of conformations around Ser53 (Fig. 4c). In particular, the carbonyl group of Ser52 shows a range of states up to $180^{\circ}$. Although this flexibility is also seen in two of the six chains of the S53A nonprocessing protein, it is more pronounced in the N47A structure.

\subsection{Mechanistic implications}

The E109Q and N47A mutant proteins were each assayed for decarboxylation activity by measuring the amount of ${ }^{14} \mathrm{CO}_{2}$ that was released from labeled ${ }^{14} \mathrm{C}$-arginine. The results of the assay revealed that both mutant proteins have reduced activity compared with the wild type (Table 2). The E109Q activity was decreased by 7.7 -fold compared with the wild type. Glu109 has been implicated in both the decarboxylation
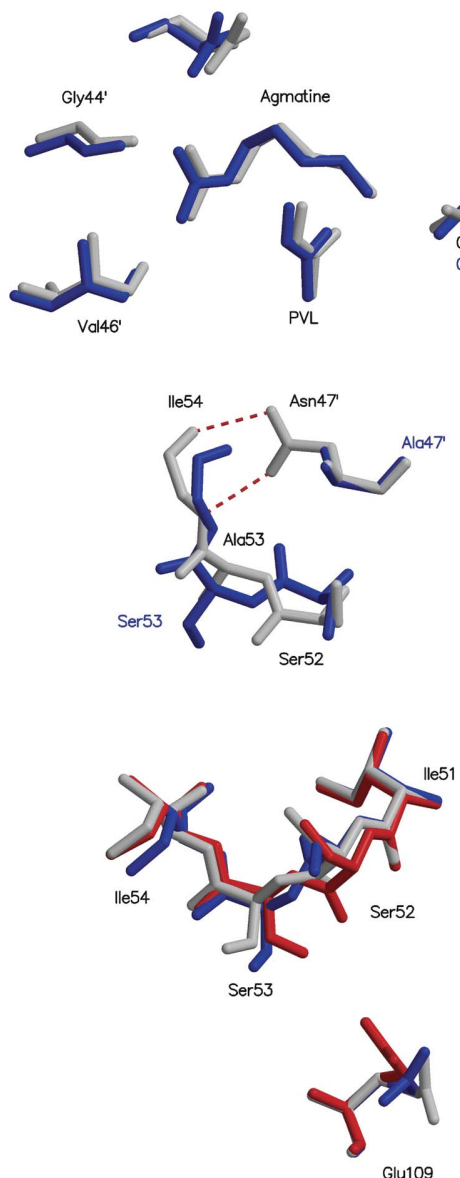

(c)

\section{Figure 4}

Active-site superpositions. (a) The E109Q (blue) mutant protein superimposed on the wildtype (gray) PvlArgDC. No significant conformational changes were observed. (b) Superposition of N47A (blue) with the unprocessed S53A mutant protein (gray). Although both mutant proteins show no processing in this chain, there is a shift in the Ile54 position in the N47A mutant, most likely owing to loss of hydrogen bonding. (c) Comparison of three protomers from one asymmetric unit of the N47A mutant protein. This mutation resulted in increased flexibility in the key residues involved in the autoprocessing reaction. In all panels residues labelled with a prime are from a second protomer.
Table 2

PvlArgDC decarboxylation activity.

\begin{tabular}{lc}
\hline & Activity $\left(\mathrm{pmol} \mathrm{min}^{-1} \mu \mathrm{g}^{-1}\right)$ \\
\hline Wild type & $1014 \pm 58$ \\
E109Q & $132 \pm 8$ \\
N47A & 2 \\
\hline
\end{tabular}

reaction and pyruvoyl formation. In the decarboxylation reaction, arginine reacts with the pyruvoyl group to form a Schiff base. The Schiff base is protonated, which stabilizes the $\alpha$-carbanion intermediate and allows decarboxylation to occur (Hackert \& Pegg, 1997). The structure of the wild-type PvlArgDC suggested that Glu109 was the acid that protonates the $\alpha$-carbanion intermediate. The activity assay showed reduced decarboxylation activity, suggesting that Glu109 may indeed be involved in the decarboxylation reaction; however, the decrease in activity is small, suggesting that other factors are involved.

In the mechanism for the formation of the pyruvoyl group, the carbonyl $\mathrm{C}$ atom of Ser52 undergoes nucleophilic attack by the side chain of Ser53. The oxyoxazolidine intermediate then undergoes a rearrangement to an ester followed by $\beta$-elimination, resulting in a new $\mathrm{C}$-terminus at Ser52 of the $\beta$ chain and dehydroalanine at the $\mathrm{N}$-terminus of the $\alpha$ chain (position 53 of the proenzyme). Elimination of $\mathrm{NH}_{3}$ results in a pyruvoyl group at the $\mathrm{N}$-terminus of the $\alpha$ chain (Hackert \& Pegg, 1997). Glu109 has been proposed to act as an acid for the formation of the ester intermediate and as a base for the $\beta$-elimination step of the mechanism. The crystal structure of E109Q reveals a different rotameric conformation of Gln109 compared with the wild-type structure; however, the pyruvoyl group is apparent and in each the product is bound to the protomer active sites. This is consistent with a reduction in activity for the E109Q mutant.

The activity of N47A was reduced by approximately 500-fold compared with the wild-type protein. Unlike Glu109, Asn47 has not been implicated in acid/base chemistry for either the decarboxylation reaction or pyruvoyl-group formation; however, we pursued this mutant protein because of the conservation of this residue both in PvlArgDCs and the structurally related pyruvoyl-dependent histidine decarboxylase. These observations suggest a structural role for Asn47. In the wild-type structure, Asn47 forms a hydrogen bond to the Ile54 main-chain $\mathrm{N}$ atom and carbonyl $\mathrm{O}$ atom. These hydrogen bonds may be 
involved in orienting Ser53, thus affecting the rate of pyruvoyl formation. The reduction in decarboxylation activity of N47A is significant. However, the crystal structure of N47A showed a mixture of processed and unprocessed states and it is likely that the reduced rate of decarboxylation activity results in part from incomplete pyruvoyl-group formation.

\subsection{Comparison to pyruvoyl-dependent L-aspartate- $a$-decarboxylase}

Although pyruvoyl-dependent L-aspartate- $\alpha$-decarboxylase (ADC) is not structurally homologous to PvlArgDC, the mechanism of pyruvoyl formation is similar. In ADC, the autoprocessing occurs between Gly24 and Ser25. The structure of wild-type unprocessed ADC has been solved to $1.95 \AA$ resolution (PDB code 1ppy; Schmitzberger et al., 2003) and revealed that the carbonyl atom of Ser25 forms a hydrogen bond to the main-chain $\mathrm{N}$ atom of Asn72. The ADC Ser25 carbonyl was in a different conformation compared with the PvlArgDC S53A structure. Interestingly, N47A PvlArgDC displays a wide range of conformations near Ser53 and all of the unprocessed chains show the Ser53 carbonyl in a conformation similar to that in the unprocessed ADC structure (Fig. 4c). The position of the Ser53 carbonyl suggests that Asn47 in PvlArgDC may be involved in hydrogen-bonding interactions with the carbonyl of Ser53 as previously suggested (Schmitzberger et al., 2003).

\section{Conclusions}

Two PvlArgDC mutant proteins were studied in order to understand the roles of Asn47 and Glu109 in pyruvoyl-group formation and decarboxylation of arginine. The E109Q mutation resulted in a fully processed enzyme in the crystal structure and decarboxylation-activity assays revealed that the E109Q mutation reduced the activity by 7.7 -fold compared with the wild-type enzyme. This modest reduction in activity suggests that Glu109 is important for the decarboxylation reaction but that other factors are involved. Given the similarity of the autoprocessing and decarboxylation reactions, Glu109 may also play a role in pyruvoyl-group formation. Attempts to determine the relative rates of the autoprocessing reaction were inconclusive; however, the mutant proteins showed little processing after $3 \mathrm{~h}$. The N47A mutation resulted in a crystal structure containing a mixture of processed and unprocessed states. Activity assays showed that the mutation resulted in an approximately 500 -fold decrease in the rate of decarboxylation compared with the wild type. This mutation resulted in a loss of hydrogen bonding to Ile54 and also a possible loss of hydrogen bonding to Ser53, creating increased flexibility at the autoprocessing site. Based on the mixture of states found in the N47A crystal structure, the reduced activity is most likely to be the consequence of a combination of unprocessed proenzyme and a reduced rate of decarboxylation.

We would like to acknowledge the A1 beamline at the Cornell High Energy Synchrotron Source supported by NIH grant RR-01646 for providing beam time. The authors would also like to acknowledge Ms Leslie Kinsland for help in preparing this manuscript.

\section{References}

Ausubel, F. M., Brent, F., Kingston, R. E., Moore, D. D., Seidman, J. G., Smith, J. A. \& Struhl, K. (1987). Current Protocols in Molecular Biology. New York: John Wiley \& Sons.

Brünger, A. T., Adams, P. D., Clore, G. M., DeLano, W. L., Gros, P., Grosse-Kunstleve, R. W., Jiang, J.-S., Kuszewski, J., Nilges, M., Pannu, N. S., Read, R. J., Rice, L. M., Simonson, T. \& Warren, G. L. (1998). Acta Cryst. D54, 905-921.

DeLano, W. L. (2002). The PyMOL User's Manual. DeLano Scientific, San Carlos, USA.

Emsley, P. \& Cowtan, K. (2004). Acta Cryst. D60, 2126-2132.

Esnouf, R. M. (1997). J. Mol. Graph. 15, 132-134.

Esnouf, R. M. (1999). Acta Cryst. D55, 938-940.

Graham, D. E., Xu, H. \& White, R. H. (2002). J. Biol. Chem. 277, 23500-23507.

Grishin, N. V. \& Phillips, M. A. (1994). Protein Sci. 3, 2455-2458.

Hackert, M. L. \& Pegg, A. E. (1997). Comprehensive Biological Catalysis, edited by M. L. Sinnott, pp. 201-216. London: Academic Press.

Jones, T. A., Zou, J.-Y., Cowan, S. W. \& Kjeldgaard, M. (1991). Acta Cryst. A47, 110-119.

Laskowski, R. A., MacArthur, M. W., Moss, D. S. \& Thornton, J. M. (1993). J. Appl. Cryst. 26, 283-291.

Merritt, E. A. \& Bacon, D. J. (1997). Methods Enzymol. 277, 505-524.

Otwinowski, Z. \& Minor, W. (1997). Methods Enzymol. 276, 307-326.

Poelje, P. D. van \& Snell, E. E. (1990). Annu. Rev. Biochem. 59, 29-59.

Recsei, P. A., Huynh, Q. K. \& Snell, E. E. (1983). Proc. Natl Acad. Sci. USA, 80, 973-977.

Sambrook, J., Fritsch, G. F. \& Maniatis, T. (1989). Molecular Cloning: A Laboratory Guide. Cold Spring Harbor, USA: Cold Spring Harbor Laboratory Press.

Sandmeier, E., Hale, T. I. \& Christen, P. (1994). Eur. J. Biochem. 221, 997-1002.

Schmitzberger, F., Kilkenny, M. L., Lobley, C. M., Webb, M. E., Vinkovic, M., Matak-Vinkovic, D., Witty, M., Chirgadze, D. Y., Smith, A. G., Abell, C. \& Blundell, T. L. (2003). EMBO J. 22, 61936204.

Tabor, C. W. \& Tabor, H. (1984). Annu. Rev. Biochem. 53, 749-790.

Tolbert, W. D., Graham, D. E., White, R. H. \& Ealick, S. E. (2003). Structure, 11, 285-294.

Williams, K. (1997). Biochem. J. 325, 289-297. 